\title{
Accuracy of perfusion MRI with high spatial but low temporal resolution to assess invasive breast cancer response to neoadjuvant chemotherapy: a retrospective study
}

Cédric de Bazelaire ${ }^{1 *}$, Raphael Calmon ${ }^{1}$, Isabelle Thomassin², Clément Brunon ${ }^{3}$, Anne-Sophie Hamy4, Laure Fournier $^{3}$, Daniel Balvay ${ }^{3}$, Marc Espié ${ }^{4}$, Nathalie Siauve ${ }^{3}$, Olivier Clément ${ }^{3}$, Eric de Kerviler ${ }^{1}$ and Charles-André Cuénod ${ }^{3}$

\begin{abstract}
Background: To illustrate that Breast-MRI performed in high spatial resolution and low temporal resolution (1 minute) allows the measurement of kinetic parameters that can assess the final pathologic response to neoadjuvant chemotherapy in breast cancer.
\end{abstract}

Methods: Breast-MRI was performed in 24 women before and after treatment. Eight series of 1.11 minute-duration were acquired with a sub-millimeter spatial resolution. Transfer constant $\left(K^{\text {trans }}\right)$ and leakage space $\left(V_{e}\right)$ were calculated using measured and theoretical Arterial Input Function (AIF). Changes in kinetic parameters after treatment obtained with both AIFs were compared with final pathologic response graded in non-responder $(<$ 50\% therapeutic effect), partial-responder (> 50\% therapeutic effect) and complete responder. Accuracies to identify non-responders were compared with receiver operating characteristic curves.

Results: With measured-AIF, changes in kinetic parameters measured after treatment were in agreement with the final pathological response. Changes in $\mathrm{V}_{e}$ and $\mathrm{K}^{\text {trans }}$ were significantly different between non- $(\mathrm{N}=11)$, partial-( $\mathrm{N}=$ 7), and complete $(N=6)$ responders, $\left(P=0.0092\right.$ and $P=0.0398$ respectively). A decrease in $V_{e}$ of more than $-72 \%$ and more than $-84 \%$ for $K^{\text {trans }}$ resulted in $73 \%$ sensitivity for identifying non-responders (specificity $92 \%$ and $77 \%$ respectively). A decrease in $V_{e}$ of more than $-87 \%$ helped to identify complete responders (Sensitivity $89 \%$, Specificity 83\%). With theoretical-AIF, changes in kinetic parameters had lower accuracy.

Conclusion: There is a good agreement between pathological findings and changes in kinetic parameters obtained with breast-MRI in high spatial and low temporal resolution when measured-AIF is used. Further studies are necessary to confirm whether MRI contrast kinetic parameters can be used earlier as a response predictor to neoadjuvant chemotherapy.

\section{Background}

Neoadjuvant chemotherapy is increasingly used in breast cancer patients to decrease the tumour size in large cancers to enable breast-conserving treatment. Accurate evaluation of the treatment response before surgery offers the potential to avoid unnecessary mutilating procedures

\footnotetext{
* Correspondence: cedric.de-bazelaire@sls.aphp.fr

${ }^{1}$ Radiologie, Hôpital Saint-Louis - Inserm U728 - Université Paris VII, 1 Avenue Claude Vellefaux, Paris, 75010, France

Full list of author information is available at the end of the article
}

in patients with a favorable prognosis, without jeopardizing local control or long-term survival. Compared with physical examination, and conventional modalities (US and mammography), breast MRI appears to be the best monitoring method for neoadjuvant chemotherapy $[1,2]$. Although MR imaging may be superior to other methods $[3,4]$, the correlation between conventional anatomic MRI analysis and histopathological response is not perfect [5]. The determination of residual tumour size is underestimated and unreliable in carcinomas significantly

\section{() Biomed Central}


responding to chemotherapy which may lead to missed detections in up to $30 \%$ of patients [6]. There is now increasing evidence that functional analysis of the microcirculation by using dynamic contrast material-enhanced MR imaging could be used to identify responders and non-responders during and/or after neoadjuvant chemotherapy more reliably than conventional anatomic MRI results alone [6-10]. The functional analysis is based on post-therapeutic changes of microvessels permeability, tissue perfusion, blood volume, and extracellular leakage space. These parameters can be obtained by analyzing the enhancement kinetics measured in the tissue of interest and in its afferent artery (Arterial Input Function, AIF) using compartmental modeling $[11,12]$

Several compartmental models can be chosen for assessment. The simplest model, applied to MRI data by both Larsson et al. [13] and Tofts and Kermode [14] allows calculation of the transfer constant $\left(\mathrm{K}^{\text {trans }}\right)$ that reflects simultaneously perfusion and permeability, and the leakage space $\left(\mathrm{V}_{\mathrm{e}}\right)$. More recent models potentially enable to distinguish perfusion and permeability separately [15-18]. The choice of the model and kinetic parameters to be calculated depends on the acquisition parameters. In particular, the temporal resolution determines whether the vascular component can be taken into account. Indeed, perfusion and blood volume measurement requires a high temporal resolution, because the sampling interval must be less than the mean transit time of the contrast agent [19], which is usually less than 2 seconds [20]. In breast MRI, high spatial resolution is required because most of the diagnostic criteria are based on lesion morphology [21]. Due to technical reasons in most current systems of magnetic field up to $1.5 \mathrm{~T}$, the $3 \mathrm{D}$ high spatial resolution limits the temporal resolution to as low as 1 minute and imposes to use the simplest model limited to the estimation of $\mathrm{K}^{\text {trans }}$ and $\mathrm{V}_{\mathrm{e}}$ values [22-24].

The estimation of the kinetic parameters requires two sets of data: the variation of contrast concentration in time in the tissue of interest and in the feeding artery (arterial input function, AIF). In MRI, concentrations can be non-invasively derived from signal intensity. In Breast MRI, the measurement of the AIF can be difficult. A large blood vessel such as the aorta is rarely included in the field of view [25] and the measurements of the internal thoracic artery can be difficult due to its small size with the risk of partial volume artifact [26]. For these reasons, several authors use a calculated theoretical AIF [7]. The time curve of contrast agent concentration in the plasma is represented by a biexponential decay based on the AIF measured by Weinmann et al [27]. However, the use of a calculated theoretical AIF may entail errors in MR estimates of kinetic parameters.

The purpose of this retrospective study is to demonstrate that even with low temporal resolution routine
MRI protocol, changes in microcirculation kinetic parameters such as $\mathrm{K}^{\text {trans }}$ and $\mathrm{V}_{\mathrm{e}}$ can be used to determine tumor response to neoadjuvant chemotherapy in breast cancer as observed at the final pathological evaluation after surgery.

\section{Methods \\ Demography}

This study was part of the Remagus [28,29] protocol, approved by the ethics committee, and requiring informed consent before enrollment from all patients. Three physicians specialized in breast cancer with over 10 years of experience performed the patients follow up and determined the clinical response to neoadjuvant chemotherapy according the WHO criteria. The Remagus protocol was a neoadjuvant chemotherapy trial for locally advanced breast cancers including 4 cycles of Antracycline and Cyclophosphamide, followed by 4 cycles of Docetaxel chemotherapy. Patients with noninflammatory, stage II to III breast cancer were included during 12 month. Diagnosis of invasive breast carcinoma was made by core needle biopsy in all patients. Surgery was performed less than 4 weeks after the last course of chemotherapy. Patients either underwent mastectomy or wide local excision with axillary lymph node dissection.

\section{Pathologic Assessment}

One senior pathologist with 20 years of experience in breast pathology blinded to the MRI results assessed tumor response and graded according to the scale established by Sataloff [30] as shown Table 1: total or near-total therapeutic effect (grade A), more than 50\% therapeutic effect but less than total or near-total effect (grade B), less than 50\% therapeutic effect but visible effect (grade C), or no therapeutic effect (grade D). Pathologic tumor regression was used as the gold standard to evaluate treatment response. For comparison with imaging five groups of patients were defined according to the response grade: complete responder group (grade A), partial responder group (grade $\mathrm{B})$, responder group $(\mathrm{A}+\mathrm{B})$, non-responder group (grade $\mathrm{C}+\mathrm{D}$ ), and non-complete responders (grades $\mathrm{B}+\mathrm{C}+\mathrm{D})$.

Table 1 Tumor response graded according to the scale established by Sataloff

\begin{tabular}{cl}
\hline $\begin{array}{c}\text { Sataloff } \\
\text { Grade }\end{array}$ & Therapeutic effect at pathology \\
\hline A & Total or near-total \\
B & More than $50 \%$ therapeutic effect but less than total or \\
& near-total effect \\
C & Less than $50 \%$ therapeutic effect but visible effect \\
D & No therapeutic effect \\
\hline
\end{tabular}




\section{MRI protocol}

All patients underwent two breast MRI, the first exam less than 1 week before the beginning of the treatment and the second MRI performed after completion of chemotherapy treatment and less than 2 weeks before surgery. All MRI were performed on a 1.5 Tesla Siemens Symphony TIM MR system (Erlangen, Germany), with a breast-specific coil with four elements, CP Breast Array Coil. DCE-MRI was acquired with a 3D T1-weighted gradient echo sequence using a TR of $4.67 \mathrm{~ms}$, a TE of $1.65 \mathrm{~ms}$ and a flip angle of $12^{\circ}$. The DCE-MRI sequences (120 contiguous 1.2 -mm-thick slices, $320 \times 280 \mathrm{~mm}$ FOV, $380 \times$ 300 matrix, 1.11 min scan duration, axial slices on both breasts) were acquired at $0,1.36,2.47,3.59,5.10,6.21$, 7.32 , and $8.44 \mathrm{~min}$. The contrast agent, gadoterate dimeglumine (Dotarem ${ }^{\circledR}$, Guerbet, France), was injected immediately after the acquisition of the first DCE-MRI sequence with an automatic injector (Spectris, Medrad, $\mathrm{UK}$ ) at a dose of $0.1 \mathrm{mmol} / \mathrm{Kg}$ with a rate of $2 \mathrm{ml} / \mathrm{sec}$ and pushed by saline serum. The minimum delay between the end of the contrast injection and the beginning of next sequence acquisition was 20 second.

\section{Data processing}

A senior and a junior radiologist with 8 years and 2 years of experience in breast imaging respectively, performed all image analysis. Tumor sizes were measured with electronic calipers on high-resolution T1 weighted post contrast images (measured on the 90-120 second subtraction image), following the WHO methodology, the same day of the exam by the same radiologist as required by the clinical protocol [31], the functional analysis was performed retrospectively by the same radiologists. For this analysis, the use of a 3D acquisition for the DCE-MRI allowed the selection of region of interest (ROI) at a different level for tumor than for internal thoracic artery when necessary. Regions of interest were drawn by the radiologist on subtraction images with an appropriate window and magnification factor to optimize the detection of both enhancing tumor margin and internal thoracic artery. For the AIF, the center of the ROIs were manually defined in the center of the internal thoracic artery in the native images at the second acquisition time (first acquisition after injection), and the 8 surrounding pixels were automatically selected by the computer. This 9 pixel square was automatically reproduced at the same position at all acquisition time. Then, each ROI was manually translated when necessary for motion artifacts correction to avoid partial volume artifacts. The tumor ROIs were placed around the edge of the anatomically defined tumor but away from non-enhancing areas which were either necrotic or so poorly perfused that they could not be evaluated in functional imaging.
With the 3D T1-weighted gradient echo sequence use for the DCE-MRI, the contrast information was recorded 30 seconds after the beginning of the acquisition and at least 50 second after the injection. Thus, we assumed that the contrast information was measured after the first pass of the bolus of contrast media in the artery and therefore after the peak concentration [32]. Hence, for all patients, contrast media concentrations in artery and tumor were supposed to be low ( $<2 \mathrm{mM}$ Gd-DOTA) and a linear relationship was assumed between signal intensity kinetics measured in ROIs and contrast media concentrations according with the following equations:

$$
\begin{aligned}
& C_{t}(t)=R \cdot\left(I S_{t}(t)-I S_{t}(0)\right) \\
& C_{p}(t)=R \cdot\left(I S_{p}(t)-I S_{p}(0)\right)
\end{aligned}
$$

Where $C_{t}$ and $C_{p}$ are the concentrations of contrast media in the tissue of interest and plasma, respectively. $\mathrm{IS}_{\mathrm{t}}$ and $\mathrm{IS}_{\mathrm{p}}$ are the signal intensities measured with the ROIs in the tissue of interest and the internal thoracic artery, respectively. $\mathrm{R}$ is an amplitude constant, which is simplified in equation 3 .

Custom software [33] written with Matlab ${ }^{\circledR}$ (MathWorks Inc., Natick, MA, USA) was used to calculate kinetic parameters. These parameters included the transfer constant, $\mathrm{K}^{\text {trans }}\left(\mathrm{min}^{-1}\right)$, of gadolinium-based contrast agent between blood plasma and the extravascular extracellular space (EES), and the EES fractional volume, $\mathrm{V}_{\mathrm{e}}(\%)$. Parameters were adjusted using the modified Kety model applied to MRI data by both Larsson et al [13] and Tofts and Kermode [34] and fully described recently by various authors such as Buckley [19] or Padhani [7]:

$$
C_{t}(t)=K^{\text {trans }} \cdot \int_{0}^{t} C_{p}(u) \cdot \exp \left(-\frac{K^{\text {trans }}}{V_{e}} \cdot(t-u)\right) \cdot
$$

This model assumes that the plasma volume is negligible. It also assumes that a short bolus injection time is used, with instant mixing and fast exchanges of all mobile protons within the tissue. A pixel by pixel analysis was used given a $K^{\text {trans }}$ and a $V_{e}$ maps. For all exams, parameters were adjusted twice: with a $C_{p}$ obtained directly from the measured AIF (ROIs selecting the internal thoracic artery); with a $C_{p}$ related to a calculated theoretical AIF. The theoretical AIF was modeled by using a biexponential decay that corresponds to the results measured by Weinmann et al [27]:

$$
C_{p}(t)=D \cdot\left(a_{1} \cdot e^{-m_{1} \cdot t}+a_{2} \cdot e^{-m_{2} \cdot t}\right)
$$

Where $\mathrm{D}$ is the injected dose of contrast agent (in millimoles per liter per kilogram of body weight), $\mathrm{a}_{1}=$ $3.99 \mathrm{~kg} / \mathrm{L}$ and $\mathrm{a}_{2}=4.78 \mathrm{~kg} / \mathrm{L}$ are two amplitude 
constants, $\mathrm{m}_{1}=0.144 \mathrm{~min}^{-1}$ and $\mathrm{m}_{2}=0.0111 \mathrm{~min}^{-1}$ are two rate constants [27].

\section{Statistical analysis}

The kinetic parameter changes measured after treatment in each group of patients defined by final pathologic findings were compared using non parametric tests (MannWhitney $\mathrm{U}$ test for two independent random samples and Kruskal-Wallis test for three independent random samples). Kinetic parameter changes of responder patients were compared to the changes of the non-responder patients. Kinetic parameter changes after treatment were compared between complete-, partial-, and non-responder patients. Eventually, the ability of the technique to distinguish the complete responders was tested. All comparisons were performed twice: once with kinetic parameters obtained with measured AIF and then with kinetic parameters estimated with theoretical AIF. The differences in tumour size changes between groups of patients were also compared with nonparametric methods.

Receiver operating characteristic (ROC) analyses of transfer constant and EES fractional volume changes were used to select threshold for the identification of responder and non-responder patients. The diagnostic performance of changes in parameters obtained with a measured or a theoretical AIF were compared by using the approach of DeLong et Clarke-Pearson [35].

Results were analyzed using a statistical software package (Analyse-it Software, Leeds, UK) with an $\alpha$ level set at $5 \%$. All results are given with a $95 \%$ confidence interval (95\% CI).

Breast tumours size changes in MRI have an accuracy varying from $25 \%$ to $93 \%$ in detecting tumor response after neoadjuvant chemotherapy [6,29-31]. We estimate that our approach will improve the accuracy from 55\% with tumor size changes to $80 \%$ using kinetic parameter changes. Thus, sample size of 24 patients was computed to provide $90 \%$ power at the overall $5 \%$ (two-sided) significance level to detect an accuracy of $80 \%$ [32].

\section{Results}

\section{Patients and Pathologic Response}

Patient and tumor characteristics are listed in Table 2. After completion of chemotherapy, within the 24 included patients a Sataloff grade A response was identified in 6 patients, a grade B response was identified in 7 patients, and a grade $\mathrm{C}+\mathrm{D}$ was identified in 11 patients.

Using a conventional cutoff value of a decrease of $50 \%$ (WHO criteria) of tumor size after treatment compared to baseline, the sensitivity, specificity, positive and negative predictive values, and accuracy of physical examination to detect non-responder were respectively 13\% (95\% CI:0.8\%,54\%), 82\% (95\% CI:48\%,97\%), 33\%, 56\% and 53\%. The performances of conventional anatomic MRI were
Table 2 Patient and Tumor Characteristics $(n=24)$

\begin{tabular}{lc}
\hline Characteristic & Number of Patients \\
\hline Age, years (mean, range) & 48 (range, 31 to 62) \\
Tumor histology & 21 \\
$\quad$ Invasive ductal carcinoma & 3 \\
$\quad$ Invasive lobular carcinoma & \\
\hline Receptor status & $17 / 4$ \\
$\quad$ Estrogen, (positive/negative) & $10 / 11$ \\
Progesterone (positive/negative) & $9 / 12$ \\
Her2/neu (positive/negative) & \\
\hline Scarff and Bloom Richardson grade & 2 \\
I & 12 \\
II & 9 \\
III & \\
\hline Stage & 2 \\
IIA & 5 \\
IIB & 7 \\
IIIA & 8 \\
\hline IIIB &
\end{tabular}

27\% (95\% CI:6\%,61\%), 77\% (95\% CI:46\%,95\%), 50\%, 56\% and $54 \%$. Physical examination and conventional morphological MRI based on size measurement were not correlated with the pathologic response after chemotherapy (Mann-Whitney $U$ test, $\mathrm{P}=0.44$ and $\mathrm{P}=0.42$ respectively).

Interestingly, physical examination and conventional morphological MRI were more accurate for the detection of complete responders when compared with theirs ability to detect non-responders, (Sensitivity, Specificity, Positive Predictive Value, Negative Predictive Value, and accuracy of 85\% (95\% CI:54\%,97\%), 67\% (95\% CI:24\%,94\%), 85\%, $67 \%$, and $79 \%$ with the physical exam and $67 \%(95 \%$ CI:41\%,87\%), 83\% (95\% CI:36\%,100\%), 92\%, 45\% and 71\% respectively in MRI). Physical examination was better correlated with the final pathological response than conventional morphological MRI in detecting complete responders (Mann-Whitney $\mathrm{U}$ test, $\mathrm{P}=0.05$ and $\mathrm{P}=0.22$ respectively).

Evaluation of the neoadjuvant chemotherapy in DCE-MRI

The selection and measurement of the internal thoracic artery was possible in all patients $(\mathrm{N}=24)$. Changes in kinetic parameters after the last course of chemotherapy were obtained using measured and theoretical AIF.

\section{With measured AIF}

Under treatment, changes in $\mathrm{K}^{\text {trans }}$ and $\mathrm{V}_{\mathrm{e}}$ were significantly different between non-responders (grade $\mathrm{C}+\mathrm{D}$ ) and responders (grades $\mathrm{A}+\mathrm{B}$ ), (Mann-Whitney $\mathrm{U}$ test, $\mathrm{P}=0.01$, $\mathrm{P}<0.01$ respectively). Moreover, changes in $\mathrm{K}^{\text {trans }}$ and $\mathrm{V}_{\mathrm{e}}$ were significantly different between non-responders (grade $\mathrm{C}+\mathrm{D}$ ), partial responders (grade $\mathrm{B}$ ) and complete responders (grade A) as seen figure 1 (Kruskal-Wallis test, 
$\mathrm{P}=0.04$ and $\mathrm{P}<0.01$ respectively). Eventually, a higher decrease of $\mathrm{V}_{e}(-90 \%$ (95\% CI:-97\%,-80\%)) was noted in complete responders (grade A) than in non-complete responders (grade $\mathrm{B}+\mathrm{C}+\mathrm{D}),(-68 \%$ (95\% CI: $-82 \%,-19 \%)$; Mann-Whitney $\mathrm{U}$ test, $\mathrm{P}<0.01)$. All results are summarized Table 3 and 4.

\section{With theoretical AIF}

$\mathrm{V}_{\mathrm{e}}$ changes were found to be different between nonresponders (grade, $\mathrm{C}+\mathrm{D}$ ) and responders (grades, $\mathrm{A}+\mathrm{B}$ ), (Mann-Whitney $\mathrm{U}$ test, $\mathrm{P}=0.05$ ). However, no significant difference was found between patient groups with $\mathrm{K}^{\text {trans }}$.

Typical examples of complete responder (\#22) and non-responder $(\# 10)$ patients are given in figure 2 and 3.

\section{Identification of responders and non-responders}

After treatment, a reduction of less than - $84 \%$ in transfer constant $\left(K^{\text {trans }}\right)$ with measured AIF, would have had a 73\% (95\% CI:39\%,94\%) sensitivity in the identification of 8 of 11 non-responders patients (i.e., pathologic subgroup $\mathrm{C}+\mathrm{D})$ and would have excluded 3 out of 13 responders (specificity 77\% (95\% CI:46\%,95\%), positive predictive value $73 \%$, negative predictive value $77 \%$, accuracy $75 \%$, area under ROC curve 0.80 (95 CI: $0.62,0.99))$. With a theoretical AIF to assess $\mathrm{K}^{\text {trans }}$, the cutoff $(-85 \%)$ had lower accuracy (sensitivity 46\% (95\% CI:167\%,76\%), specificity 54\% (95\% CI:25\%,80\%), positive predictive value $46 \%$, negative predictive value $54 \%$, accuracy 50\%, area under ROC curve 0.48 (95\% CI: $24 \%, 73 \%)$ ). However, the difference between areas under ROC curves of $K^{\text {trans }}$ obtained with measured and theoretical AIF (Figure 4) was not significantly different (Clarke-Pearson test, $\mathrm{P}=0.1475$ ).

A reduction of less than $-72 \%$ in leakage space $\left(V_{e}\right)$ with measured AIF, would have enabled identification of 8 of eleven non-responders (sensitivity 73\% (95\%

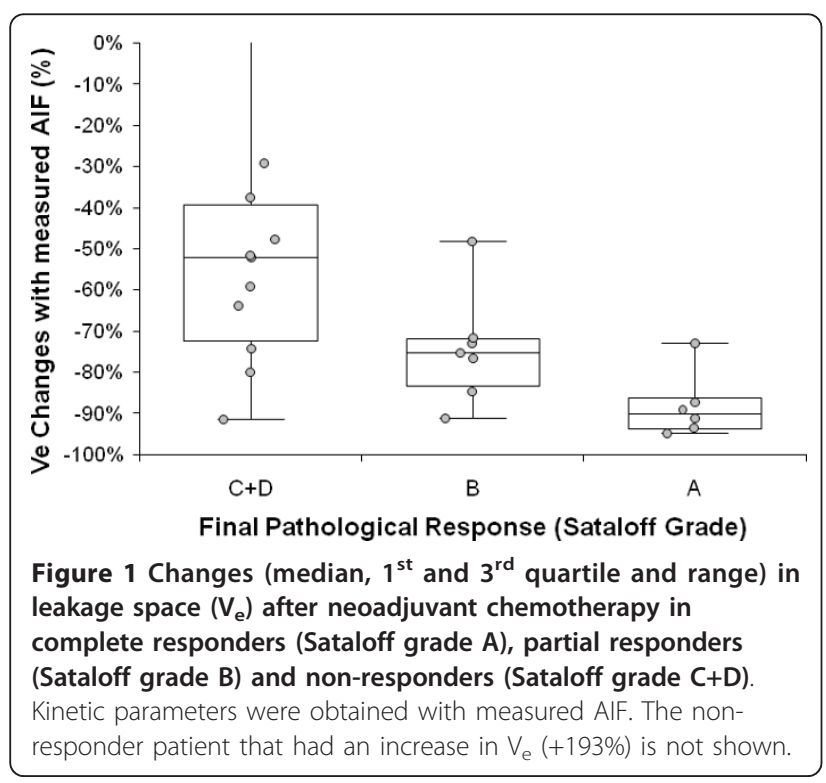

CI:39\%,94\%)) and would have excluded only 1 out of 13 responders (specificity 92\% (95\% CI:64\%,100\%)), positive predictive value $89 \%$, negative predictive value $80 \%$, accuracy $83 \%$, area under ROC curve 0.83 (95\% CI: $64 \%, 100 \%)$ ). With a theoretical AIF to assess $\mathrm{V}_{\mathrm{e}}$, the cutoff $(-51 \%)$ had lower accuracy (sensitivity 64\% (95\% CI:31\%,89\%), specificity 100\% (95\% CI:75\%,100\%), positive predictive value $100 \%$, negative predictive value $77 \%$, accuracy $83 \%$, area under ROC curve 0.74 (95\% CI:51\%,97\%)). However, the difference between areas under ROC curves of $\mathrm{V}_{\mathrm{e}}$ obtained with measured and theoretical AIF (Figure 4) was not significantly different (Clarke-Pearson test, $\mathrm{P}=0.3216$ ). Moreover, a reduction of less than $-87 \%$ in $V_{e}$ with measured AIF, would have enabled identification of 5 of 6 complete responders and 16 of 18 non complete responders (sensitivity 89\% (95\% CI:65\%,99\%), specificity 83\% (95\% CI:36\%,100\%), positive predictive value $94 \%$, negative predictive value $71 \%$, accuracy $88 \%$, area under the ROC curve $87 \%$ (95\% CI:71\%,100\%)).

The accuracy of the classification between responders and non-responders was slightly improved when $\mathrm{K}^{\text {trans }}$ and $\mathrm{V}_{\mathrm{e}}$ were used jointly. With a cutoff of $-82 \%$ for $\mathrm{K}^{\text {trans }}$ and $-72 \%$ for $V_{e}$, all non-responders were distinguished (sensitivity 100\% (95\% CI:68\%,99\%)), whereas 4 out of 13 responders were misdiagnosed (specificity 69\% (95\% CI:39\%,90\%), positive predictive value $73 \%$, negative predictive value $100 \%$, accuracy $83 \%$ ).

\section{Discussion}

This clinical study have examined the ability of conventional breast DCE-MRI performed at $1.5 \mathrm{~T}$ with high spatial resolution (sub millimeter) and low temporal resolution (1 acquisition per minute), to provide changes in kinetic parameters that agree with pathological analysis of response to neoadjuvant chemotherapy. Changes in transfer constant and in leakage space obtained with a measured AIF were significantly different between the three pathologic response categories (Kruskal-Wallis test, $\mathrm{P}<0.04)$. We found that changes in the kinetic parameters correlated pathologic response to neoadjuvant chemotherapy $(\mathrm{P}<0.04)$, whereas both change in MRI-derived tumor size and final clinical examination following neoadjuvant chemotherapy failed to correlate with final pathologic response (Kruskal-Wallis test, P > 0.4 ), when complete responders and partial responders are considered. It is well recognized that size change is an imperfect assessment method for assessing the effects of neoadjuvant chemotherapy. We found an appreciable discordance between final clinical and final pathologic response in our patient group, with almost a half of clinical responders (13 of 24) failing to obtain a pathologic response. This discrepancy between clinical and pathologic response has been described by others. The 
Table 3 Changes in kinetic parameters and tumor size in 24 patients after systemic neoadjuvant chemotherapy

\begin{tabular}{|c|c|c|c|c|c|c|c|}
\hline \multirow[t]{2}{*}{ Patient \# } & \multicolumn{2}{|c|}{$\mathrm{K}^{\text {trans }}$ changes } & \multicolumn{2}{|c|}{$\mathrm{V}_{\mathrm{e}}$ changes } & \multirow{2}{*}{$\begin{array}{c}\text { Size changes } \\
\text { N/A }\end{array}$} & \multirow{2}{*}{$\begin{array}{c}\text { Clinical } \\
\text { Response }\end{array}$} & \multirow{2}{*}{$\begin{array}{c}\text { Final pathologic finding } \\
\text { Sataloff Grade }\end{array}$} \\
\hline & AIF theoretical & AIF measured & AIF theoretical & AIF measured & & & \\
\hline 1 & $-70 \%$ & $-23 \%$ & $-73 \%$ & $-80 \%$ & $0 \%$ & PR & C \\
\hline 2 & $-35 \%$ & $-20 \%$ & $-4 \%$ & $-52 \%$ & $57 \%$ & SD & C \\
\hline 3 & $-2 \%$ & $108 \%$ & $-89 \%$ & $-91 \%$ & $-100 \%$ & PR & C \\
\hline 4 & $-71 \%$ & $-22 \%$ & $-46 \%$ & $-48 \%$ & $-84 \%$ & SD & C \\
\hline 5 & $223 \%$ & $166 \%$ & $-76 \%$ & $-74 \%$ & $-100 \%$ & $P R$ & C \\
\hline 6 & $-97 \%$ & $-84 \%$ & $23 \%$ & $-29 \%$ & $158 \%$ & PR & C \\
\hline 7 & $-85 \%$ & $-67 \%$ & $-78 \%$ & $-64 \%$ & $-72 \%$ & $P R$ & C \\
\hline 8 & $-93 \%$ & $93 \%$ & $-15 \%$ & $-52 \%$ & $-99 \%$ & $C R$ & C \\
\hline 9 & $-98 \%$ & $-98 \%$ & $-50 \%$ & $-38 \%$ & $-65 \%$ & $P R$ & $\mathrm{D}$ \\
\hline 10 & $-90 \%$ & $-82 \%$ & $-50 \%$ & $-59 \%$ & $-100 \%$ & PR & C \\
\hline 11 & $-97 \%$ & $-89 \%$ & $-49 \%$ & $193 \%$ & $-62 \%$ & PR & C \\
\hline Median $(C+D)$ & $-85 \%$ & $-23 \%$ & $-50 \%$ & $-52 \%$ & $-72 \%$ & & \\
\hline $95 \mathrm{Cl}$ & [-110\%,17\%] & {$[-72 \%, 51 \%]$} & {$[-69 \%,-23 \%]$} & {$[-88 \%, 17 \%]$} & {$[-98 \%, 13 \%]$} & & \\
\hline 12 & $-85 \%$ & $-95 \%$ & $-61 \%$ & $-73 \%$ & $-71 \%$ & $C R$ & $B$ \\
\hline 13 & $-99 \%$ & $-86 \%$ & $-65 \%$ & $-77 \%$ & $-73 \%$ & $P R$ & B \\
\hline 14 & $-54 \%$ & $-89 \%$ & $-57 \%$ & $-48 \%$ & $-100 \%$ & SD & B \\
\hline 15 & $-94 \%$ & $-95 \%$ & $-75 \%$ & $-75 \%$ & $-33 \%$ & SD & B \\
\hline 16 & $65 \%$ & $-52 \%$ & $-68 \%$ & $-72 \%$ & $-100 \%$ & PR & B \\
\hline 17 & $-96 \%$ & $-84 \%$ & $-72 \%$ & $-85 \%$ & $-91 \%$ & PR & B \\
\hline 18 & $-97 \%$ & $-97 \%$ & $-77 \%$ & $-91 \%$ & $-29 \%$ & PR & B \\
\hline Median (B) & $-94 \%$ & $-89 \%$ & $-68 \%$ & $-75 \%$ & $-73 \%$ & & \\
\hline $95 \mathrm{Cl}$ & {$[-121 \%,-10 \%]$} & {$[-100 \%,-71 \%]$} & {$[-75 \%,-61 \%]$} & {$[-87 \%,-62 \%]$} & {$[-98 \%,-44 \%]$} & & \\
\hline Median $(B+C+D)$ & $-88 \%$ & $-83 \%$ & $-63 \%$ & $-68 \%$ & $-73 \%$ & & \\
\hline $95 \mathrm{Cl}$ & {$[-95 \%,-14 \%]$} & {$[-80 \%, 0 \%]$} & {$[-69 \%,-40 \%]$} & {$[-82 \%,-19 \%]$} & {$[-87 \%,-20 \%]$} & & \\
\hline 19 & $0 \%$ & $27 \%$ & $-89 \%$ & $-89 \%$ & $-40 \%$ & $P R$ & $A$ \\
\hline 20 & $-15 \%$ & $-91 \%$ & $-62 \%$ & $-95 \%$ & $-99 \%$ & $C R$ & A \\
\hline 21 & $4 \%$ & $-54 \%$ & $-89 \%$ & $-91 \%$ & $-100 \%$ & $C R$ & A \\
\hline 22 & $-29 \%$ & $-98 \%$ & $-51 \%$ & $-73 \%$ & $-100 \%$ & $C R$ & A \\
\hline 23 & $-99 \%$ & $-100 \%$ & $-95 \%$ & $-94 \%$ & $-96 \%$ & $C R$ & A \\
\hline 24 & $-94 \%$ & $-99 \%$ & $-75 \%$ & $-87 \%$ & $-97 \%$ & $P R$ & A \\
\hline Median (A) & $-22 \%$ & $-94 \%$ & $-82 \%$ & $-90 \%$ & $-98 \%$ & & \\
\hline $95 \mathrm{Cl}$ & {$[-87 \%, 10 \%]$} & {$[-122 \%,-16 \%]$} & {$[-95 \%,-59 \%]$} & {$[-97 \%,-80 \%]$} & {$[-114 \%,-64 \%]$} & & \\
\hline
\end{tabular}

Final pathologic findings were graded according to the scale established by Sataloff: complete responder group (grade A), partial responder group (grade B), responder group $(A+B)$, and non-responder group (grade $C+D)$. Surgery performed after neoadjuvant chemotherapy included mastectomy or conservative treatment or inadequate conservative surgery followed by mastectomy. $\mathrm{SD}=$ stable disease, $\mathrm{PR}=$ partial responder, $\mathrm{CR}=$ complete responder.

Table 4 Statistical results ( $P$-value) of the non parametrical test (* Kruskal-Wallis and $\neq$ Mann-Whitney $U$ test) used to compare changes in the kinetic parameters, tumor size in MRI, and clinical findings, between groups of patients defined by final pathologic findings (Sataloff Grade)

\begin{tabular}{|c|c|c|c|c|c|c|}
\hline \multirow[b]{2}{*}{$\begin{array}{l}\text { Sataloff } \\
\text { Grade }\end{array}$} & \multicolumn{2}{|c|}{$\mathrm{K}^{\text {trans }}$} & \multicolumn{2}{|c|}{$\mathrm{V}_{\mathrm{e}}$} & \multirow{2}{*}{$\begin{array}{c}\begin{array}{c}\text { Size in } \\
\text { MRI }\end{array} \\
p \text {-value }\end{array}$} & \multirow{2}{*}{$\begin{array}{c}\begin{array}{c}\text { Clinical } \\
\text { findings }\end{array} \\
p \text {-value }\end{array}$} \\
\hline & $\begin{array}{c}\text { AIF theoretical } p \text { - } \\
\text { value }\end{array}$ & $\begin{array}{c}\text { AIF measured } p \text { - } \\
\text { value }\end{array}$ & $\begin{array}{c}\text { AIF theoretical } p \text { - } \\
\text { value }\end{array}$ & $\begin{array}{c}\text { AIF measured } p \text { - } \\
\text { value }\end{array}$ & & \\
\hline $\begin{array}{c}A \text { vs } B \text { vs } C \\
+D\end{array}$ & 0,5467 & 0,0398 & 0,0799 & 0,0092 & 0,4643 & 0,4643 \\
\hline$A+B$ vs $C+D$ & 0,9095 & 0,0107 & 0,0474 & 0,0059 & 0,4244 & 0,4421 \\
\hline$A$ vs $B+C+D$ & 0,3173 & 0,1096 & 0,0532 & 0,0077 & 0,2244 & 0,0462 \\
\hline
\end{tabular}



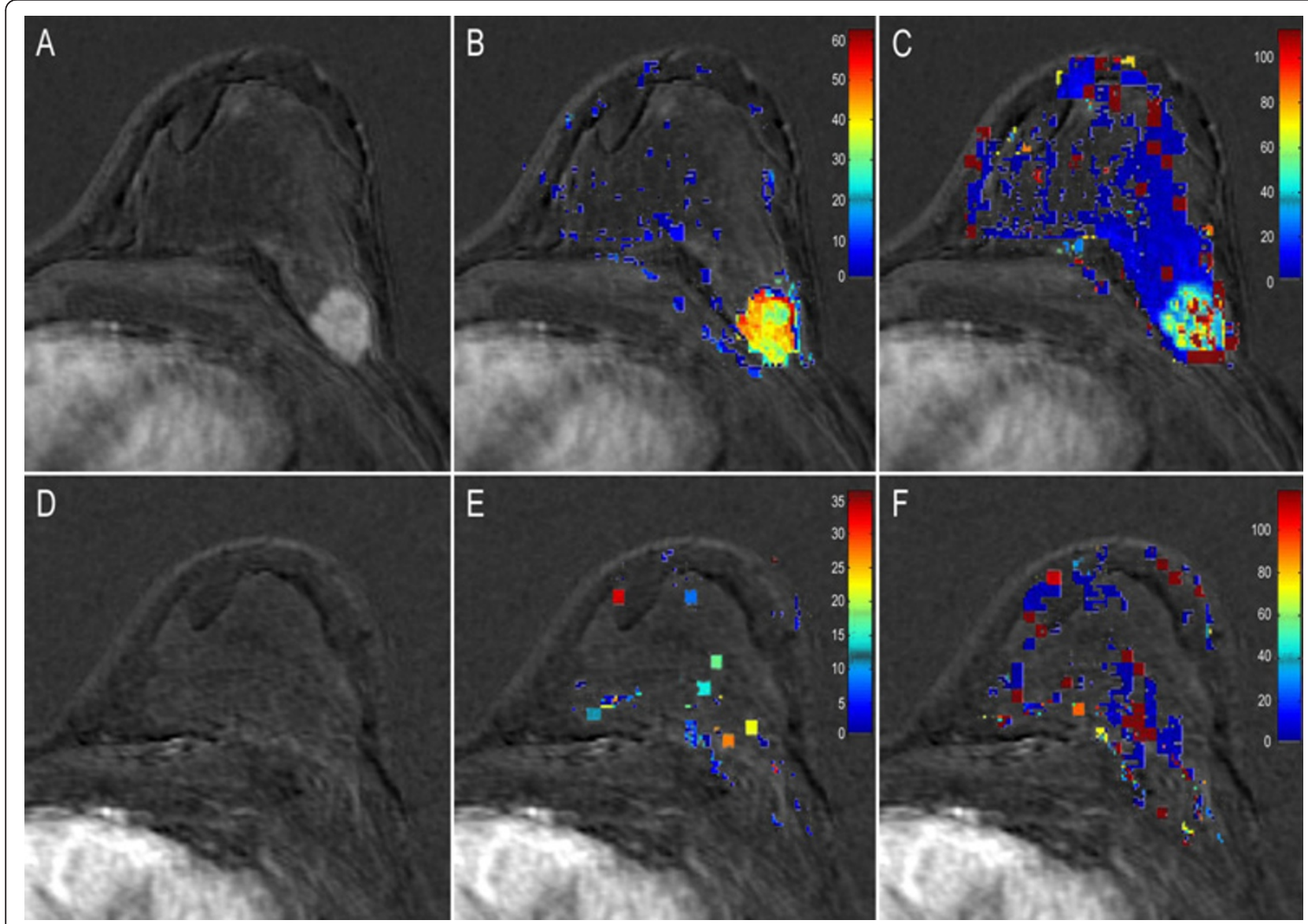

Figure 2 Images show changes in transfer constant $\left(K^{\text {trans }}\right)$ in patient 22, complete responder to neoadjuvant chemotherapy (Sataloff A). Columns show in A and D: anatomic subtraction images; in B and E: corresponding $K^{\text {trans }}$ map acquired using measured Arterial Input Function (AIF); and in C and F: corresponding $K^{\text {trans }}$ map acquired using theoretical AIF. Images A, B, and C show data before neoadjuvant chemotherapy treatment and images D, F, and G are post-treatment. After treatment a decrease of - $98 \%$ is seen in $K^{\text {trans }}$ using measured AlF values and a decrease of $-29 \%$ using theoretical AIF values. Note the difference between $\mathrm{K}^{\text {trans }}$ values before treatment when using measured and theoretical AIFs. To increase visibility of the color encoded $K^{\text {trans }}$ pixels the scale was reduced in postchemotherapy images.

NSABP-B18trial [36] showed that of the 682 patients, who received neoadjuvant chemotherapy, 247 achieved a clinical Complete Response but only 88 of these had a pathologic Complete Response.

The microvascular pressures differ from one tumor to another depending upon the vascular architecture, viscous resistance offered to blood flow and interstial fluid diffusion [37-39]. The high interstitial fluid pressure (IFP) seen in tumor without treatment would result in a low diffusion of the contrast agent in the interstitial space and hence low measured $V_{e}$ value. In responder, we can assume a decrease of the proportion of immature vessel that yield to a reduction of the IFP and an increase of $\mathrm{V}_{\mathrm{e}}$. On the other hand, tumor resistance to chemotherapy would result in ongoing production of angiogenic factors that maintain or increase the IFP and $V_{\mathrm{e}}$.

We have observed that changes in kinetic parameters obtained in low temporal resolution are more accurate when AIF are measured instead of using a calculated theoretical AIF as usually performed in the literature $[20,40]$. In this study, the comparison of ROC curves obtained for $\mathrm{K}^{\text {trans }}$ changes with a measured and a theoretical AIF shows the inability to distinguish responders and non-responder when a theoretical AIF is used. The ROC curves obtained for $V_{e}$ changes shows slight improvement in performance to distinguish responders and non-responders, not statistically significant, when measured AIF is used. Wedam et al. have evaluated the effect of Bevacizumab, an antiangiogenic treatment by using the dynamic contrast enhancement MRI with theoretical AIF [41]. The kinetic parameters $\left(K^{\text {trans }}, \mathrm{k}_{\mathrm{ep}}\right.$, and $\mathrm{v}_{\mathrm{e}}$ ) were significantly decreased after the first cycle of treatment. However, there was no significant difference in any of the DCE-MRI parameters between clinical responders and non-responders. The use of a calculated theoretical AIF may have reduced the observed decrease 


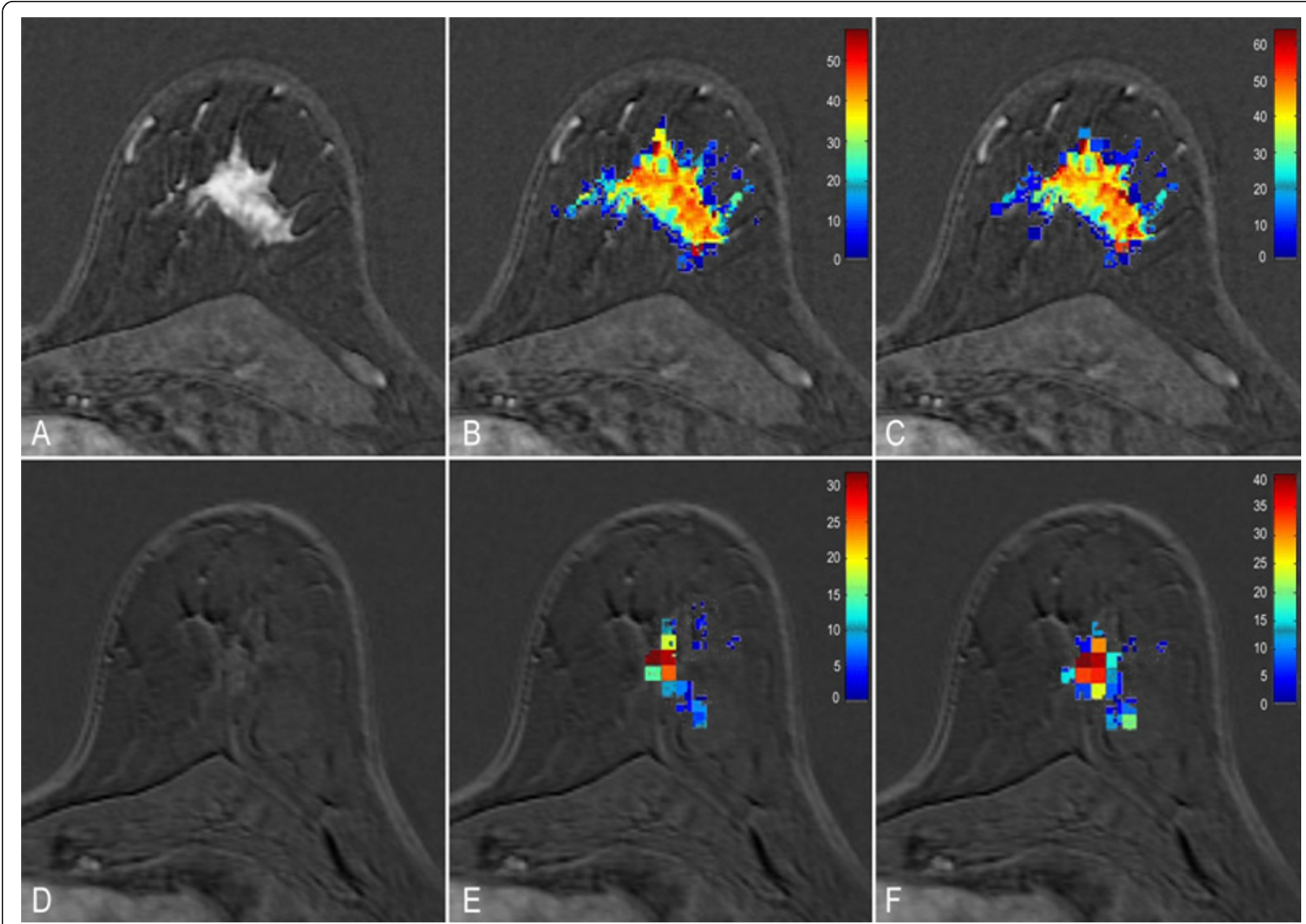

Figure 3 Images show changes in volume leakage $\left(V_{e}\right)$ in patient 10 non responder to neoadjuvant chemotherapy (Sataloff grade $C$ ) Columns show in $A$ and $D$ : anatomic subtraction images; in $B$ and $E$ : corresponding $V_{e}$ map acquired using measured Arterial Input Function (AIF); and in C and F: corresponding $V_{e}$ map acquired using theoretical AlF. Images A, B, and C show data before neoadjuvant chemotherapy treatment and images $D, F$, and $G$ are post-treatment. After treatment, low decreases in $V_{e}$ median were seen using the measured AlF (-59\%) and theoretical AIF (-50\%) in agreement with the pathological observation. Note the disagreement with tumour size changes (-100\%). To increase visibility of the color encoded $V_{e}$ pixels the scale was reduced in postchemotherapy images.

in all parameters under treatment and prevented the identification of responders. We suggest that the actual measure of the AIF be used for the calculations of $\mathrm{K}^{\text {trans }}$ and $\mathrm{v}_{\mathrm{e}}$ when data are obtained in low temporal resolution.

In this study, the selection of the Arterial Input function from the internal thoracic artery was possible in all cases, despite the risk of partial volume artifact due to the small size of the artery [26]. The use of high spatial resolution images helped to identify the internal thoracic artery. Moreover, the low temporal resolution has made possible to manually adjust ROIs on each images to encompass motion artifact and to achieve successfully the AIF in any of the cases. However, computed assisted ROI selections are suitable to achieve results with a lesser time consuming technique [42-44].

Although, the optimal temporal resolution seems to be less than 20 seconds for tracer kinetics modeling [45-47], several kinetic parameters studies with limited temporal resolution obtained with the Tofts model have been proved useful $[22,48]$. Li et al. have investigated the heterogeneity in the angiogenic response of human breast cancer xenograft to a novel angiogenesis inhibitor. They used the kinetic parameters provided by the Tofts and Kermode model with data obtained in DCE-MRI with a time resolution of 63 seconds. In their study, histogram segmentation showed that changes in the number of voxels within certain segments of the transfer constant histogram were the most sensitive variable for separating control from treated tumors. Planey et al. showed good correlation between $\mathrm{K}^{\text {trans }}$ and $\mathrm{V}_{\mathrm{e}}$ estimates from data acquired at 16.4-second temporal resolution compared to 33 and 64 second [49]. However, continuous technical improvements observed in MRI, may resolve the dilemma between the diverging demands of high temporal resolution and high spatial resolution. With the 

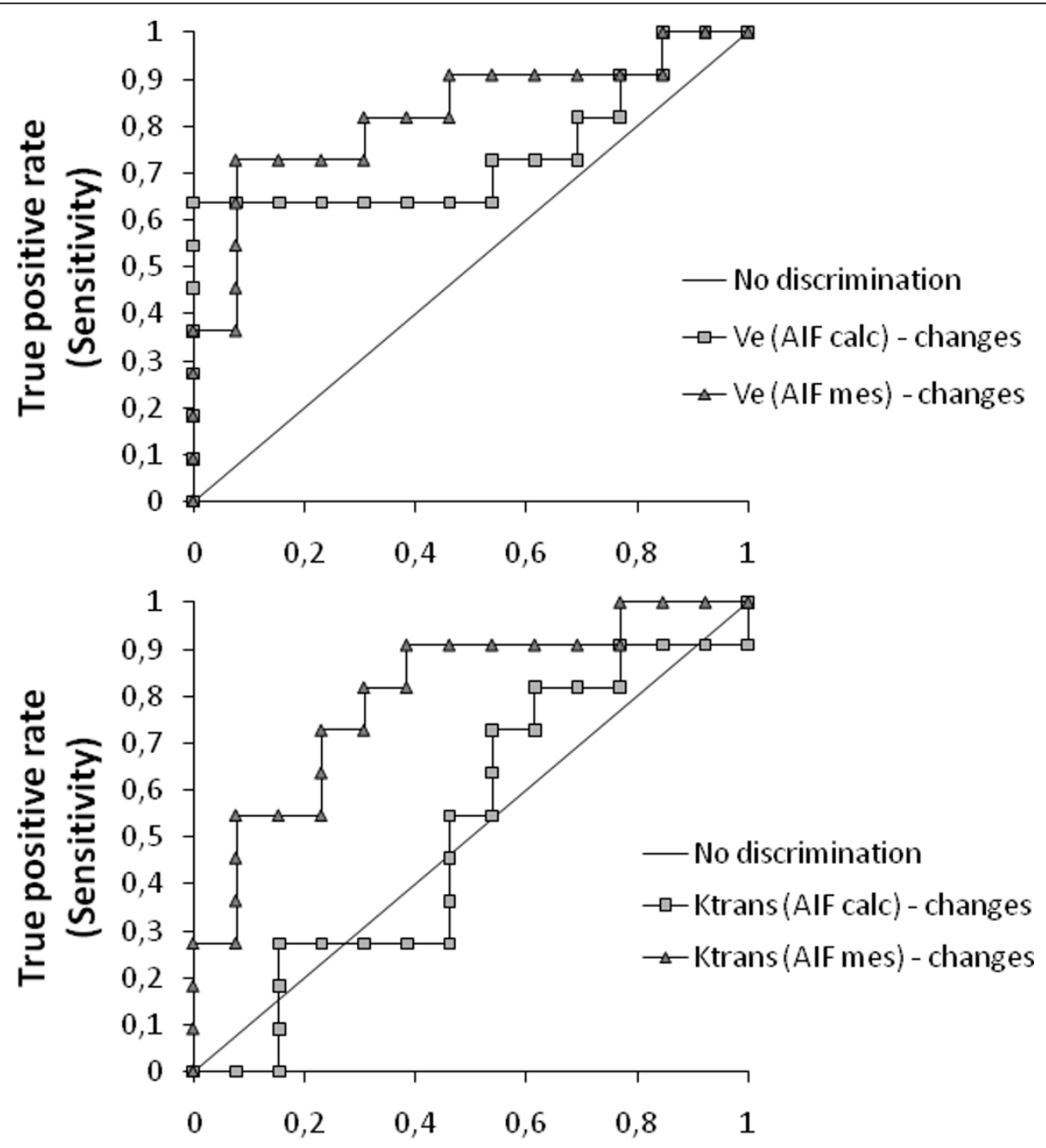

\section{False positive rate ( 1 - Specificity)}

Figure 4 ROC analysis to differentiate patients' response to neoadjuvant chemotherapy. Using measured AlF a decrease in $V_{e}$ of less than $-72 \%$ results in $73 \%$ sensitivity for identifying non-responders (specificity $92 \%$; area 0.83 ). Using theoretical AlF, the cutoff value of $-51 \%$ had lower accuracy (sensitivity 64\%; specificity 100\%; area 0.74). For transfer constant using measured AlF, a decrease of less than $-84 \%$ results in $73 \%$ sensitivity in the identification of 8 of 11 non-responders patients (Specificity 77\%; area under ROC curve 0.80). Using calculated AIF, the cutoff value of $-85 \%$ had lower accuracy (sensitivity $46 \%$; specificity $54 \%$; area under ROC curve 0.48 ).

advent of new sequences, parallel imaging and the move to higher field strengths 3.0 a temporal resolution of 13 sec with an isotropic voxel size of $1.7 \mathrm{~mm}$ is feasible [50]. Also, new multichannel breast coil may help to accelerate sequences allowing better images sampling and faster T1 mapping for an accurate signal conversion into concentrations. In this study, all non-responders were successfully classified by combining $\mathrm{K}^{\text {trans }}$ and $\mathrm{V}_{\mathrm{e}}$, whereas the separate analysis had lower sensitivity and specificity. These results incite to obtain more parameters from functional studies, which require faster sequences to accurately predict tumors response to chemotherapy.

It is clear that concentration assessment improves the final accuracy of the kinetic parameters [51]. However, this method requires accurate measurements of the tissue $\mathrm{T} 1$ relaxation time before and after contrast injection that is usually performed in few slices due to the temporal resolution constraints [52]. Nevertheless, the effect of the native tissue $\mathrm{T} 1$ relaxation on signal enhancement ratio and $\mathrm{K}^{\text {trans }} / \mathrm{V}_{\mathrm{e}}$ is very small in conditions observed in this study: short $\mathrm{TR}<10 \mathrm{~ms}$, short $\mathrm{TE}<\mathrm{T} 2^{*}$, and low dose of Gd-DTPA administration [22,34]. With a temporal resolution of 1.11 minutes, the analysis of the AIF was limited to the decay phase after the first pass, in this study. Concentrations in artery was expected to be low, and a linear relationship between concentration and signal intensity was assumed [25,32]. These assumption seems acceptable since changes in $\mathrm{K}^{\text {trans }}$ and $\mathrm{V}_{\mathrm{e}}$ achieved 
with measured AIF allowed to distinguish responders from non-responders (grade, $\mathrm{C}+\mathrm{D}$ ) (Mann-Whitney U test, $\mathrm{P}<=0.01$ ). Moreover, changes in $\mathrm{V}_{\mathrm{e}}$ were found helpful to distinguish complete responders from noncomplete responders (Mann-Whitney $\mathrm{U}$ test, $\mathrm{P}<0.01$ ). And hence, a $V_{e}$ change threshold may be defined to corroborate breast-conserving surgery in clinical ambiguous patients. All these technical limitations do not allow us to measure the absolute $K^{\text {trans }}$ and $V_{e}$ values but only relative changes in these kinetic parameters in one tumor between two exams. Though both $\mathrm{K}^{\text {trans }}$ and $\mathrm{V}_{\mathrm{e}}$ changes could be used to differentiate responders and nonresponders, a few non responder patients showed an unexpected large decrease in $\mathrm{K}^{\text {trans }}$. This could be explained by the antivascular effects of chemotherapy $[21,53-56]$ that would alter the microcirculation parameters $\left(\mathrm{K}^{\text {trans }}\right)$ before its effects can be seen in malignant tissue represented by $\mathrm{V}_{\mathrm{e}}$.

Another limitation of this preliminary study is the small number of patients included. Larger groups of patients can be studied by greater number of radiologists in furthers studies as well as more kinetic parameters with advances in medical imaging (MRI) technology. However, while conventional breast MRI had an accuracy as low as $54 \%$, with a measured AIF we have reached accuracies greater than $80 \%$ when a threshold of $-72 \%$ for $\mathrm{V}_{e}$ changes or when combined kinetic parameter changes were used in detecting residual breast cancer after neoadjuvant chemotherapy. The inclusion of 24 patients yielded a power greater than $90 \%$ to evaluate the response with these accuracies [57]. Recent studies have demonstrated the interest of diffusion and spectroscopy data in the evaluation of breast cancers response to neo adjuvant chemotherapy $[58,59]$. These parameters could be combined with kinetics parameters [8] and biological data such as hormonal and HER2 receptors status to provide a multimodality comprehensive analysis.

\section{Conclusion}

This study shows that it is feasible to assess tumours' microcirculatory kinetic parameters changes with current breast MRI protocols used in daily clinical practice. These changes in parameters are more accurate when obtained using a measured AIF, and may prove helpful to better determine breast cancer response to neoadjuvant chemotherapy than MRI based on tumor size measurements.

\section{Abbreviations}

$R O I$ : region of interest; $K^{\text {trans }}$ : inflow rate constant; $V_{\mathrm{e}}$ : leakage space; DCEMRI: dynamic contrast-enhanced MRI; MRI: magnetic resonance imaging; AIF: arterial input function; ROC: receiver operating curve characteristic; TE: echo time; TR: repetition time; VEGF: vascular endothelial growth factor; 95 Cl: 95\% confidence interval.

\section{Acknowledgements}

The authors wish to thank Michel Bruynooghe for assistance with manuscript preparation.

\section{Author details}

${ }^{1}$ Radiologie, Hôpital Saint-Louis - Inserm U728 - Université Paris VII, 1 Avenue Claude Vellefaux, Paris, 75010, France. ${ }^{2}$ Radiologie, Hôpital Tenon - Inserm U970 - Université Paris VI, 4 rue de la chine, Paris, 75020, France. ${ }^{3}$ Radiologie, Hôpital Européen George Pompidou - Inserm U970 - Université Paris V, 56 Rue Leblanc, Paris, 75015, France. ${ }^{4}$ Centre des maladies du sein, Hôpital Saint-Louis - Université Paris 7, 1 Avenue Claude Vellefaux, Paris, 75015, France.

\section{Authors' contributions}

The study was conceived by CDB, LF, NS. CDB, CB, DB and RC analyzed the data and contributed to manuscript preparation. CDB, ITN and ASH carried out the MRI experiments. CAC, EDK, OC and ME were involved in drafting the final manuscript.

All authors read and approved the final manuscript.

\section{Competing interests}

The authors declare that they have no competing interests.

Received: 31 January 2011 Accepted: 19 August 2011

Published: 19 August 2011

\section{References}

1. Kuhl C: The current status of breast MR imaging. Part I. Choice of technique, image interpretation, diagnostic accuracy, and transfer to clinical practice. Radiology 2007, 244(2):356-378.

2. Yeh E, Slanetz P, Kopans DB, Rafferty E, Georgian-Smith D, Moy L, Halpern E, Moore R, Kuter I, Taghian A: Prospective comparison of mammography, sonography, and MRI in patients undergoing neoadjuvant chemotherapy for palpable breast cancer. AJR Am J Roentgenol 2005, 184(3):868-877.

3. Partridge SC, Gibbs JE, Lu Y, Esserman LJ, Tripathy D, Wolverton DS, Rugo HS, Hwang ES, Ewing CA, Hylton NM: MRI measurements of breast tumor volume predict response to neoadjuvant chemotherapy and recurrence-free survival. AJR Am J Roentgenol 2005, 184(6):1774-1781.

4. Loo CE, Teertstra HJ, Rodenhuis S, van de Vijver MJ, Hannemann J, Muller SH, Peeters MJ, Gilhuijs KG: Dynamic contrast-enhanced MRI for prediction of breast cancer response to neoadjuvant chemotherapy: initial results. AJR Am J Roentgenol 2008, 191(5):1331-1338.

5. Hattangadi J, Park C, Rembert J, Klifa C, Hwang J, Gibbs J, Hylton N: Breast stromal enhancement on MRI is associated with response to neoadjuvant chemotherapy. AJR Am J Roentgenol 2008, 190(6):1630-1636.

6. Rieber A, Brambs HJ, Gabelmann A, Heilmann V, Kreienberg R, Kuhn T: Breast MRI for monitoring response of primary breast cancer to neoadjuvant chemotherapy. Eur Radiol 2002, 12(7):1711-1719.

7. Padhani AR, Hayes C, Assersohn L, Powles T, Makris A, Suckling J, Leach MO, Husband JE: Prediction of clinicopathologic response of breast cancer to primary chemotherapy at contrast-enhanced MR imaging: initial clinical results. Radiology 2006, 239(2):361-374.

8. Yankeelov TE, Lepage M, Chakravarthy A, Broome EE, Niermann K, Kelley MC, Meszoely I, Mayer IA, Herman CR, McManus K, Price RR, Gore JC: Integration of quantitative DCE-MRI and ADC mapping to monitor treatment response in human breast cancer: initial results. Magn Reson Imaging 2007, 25(1):1-13.

9. Delille JP, Slanetz PJ, Yeh ED, Kopans DB, Garrido L: Breast cancer: regional blood flow and blood volume measured with magnetic susceptibilitybased MR imaging-initial results. Radiology 2002, 223(2):558-565.

10. El Khoury C, Servois V, Thibault F, Tardivon A, Ollivier L, Meunier M, Allonier C, Neuenschwander S: MR quantification of the washout changes in breast tumors under preoperative chemotherapy: feasibility and preliminary results. AJR Am J Roentgenol 2005, 184(5):1499-1504.

11. Padhani AR: Dynamic contrast-enhanced MRI in clinical oncology: current status and future directions. J Magn Reson Imaging 2002, 16(4):407-422.

12. Liu Y, Bellomi M, Gatti G, Ping X: Accuracy of computed tomography perfusion in assessing metastatic involvement of enlarged axillary lymph nodes in patients with breast cancer. Breast Cancer Res 2007, 9(4):R40. 
13. Larsson HB, Stubgaard M, Frederiksen $J L$, Jensen M, Henriksen $O$, Paulson OB: Quantitation of blood-brain barrier defect by magnetic resonance imaging and gadolinium-DTPA in patients with multiple sclerosis and brain tumors. Magn Reson Med 1990, 16(1):117-131.

14. Tofts PS, Kermode AG: Measurement of the blood-brain barrier permeability and leakage space using dynamic MR imaging. 1. Fundamental concepts. Magn Reson Med 1991, 17(2):357-367.

15. Brix G, Bahner ML, Hoffmann U, Horvath A, Schreiber W: Regional blood flow, capillary permeability, and compartmental volumes: measurement with dynamic CT-initial experience. Radiology 1999, 210(1):269-276.

16. St Lawrence KS, Lee TY: An adiabatic approximation to the tissue homogeneity model for water exchange in the brain: I. Theoretical derivation. J Cereb Blood Flow Metab 1998, 18(12):1365-1377.

17. St Lawrence KS, Lee TY: An adiabatic approximation to the tissue homogeneity model for water exchange in the brain: II. Experimental validation. J Cereb Blood Flow Metab 1998, 18(12):1378-1385.

18. de Bazelaire C, Siauve N, Fournier L, Frouin F, Robert P, Clement O, de Kerviler E, Cuenod CA: Comprehensive model for simultaneous MRI determination of perfusion and permeability using a blood-pool agent in rats rhabdomyosarcoma. Eur Radiol 2005, 15(12):2497-2505.

19. Buckley DL: Uncertainty in the analysis of tracer kinetics using dynamic contrast-enhanced T1-weighted MRI. Magn Reson Med 2002, 47(3):601-606.

20. Ah-See ML, Makris A, Taylor NJ, Harrison M, Richman Pl, Burcombe RJ, Stirling JJ, d'Arcy JA, Collins DJ, Pittam MR, Ravichandran D, Padhani AR: Early changes in functional dynamic magnetic resonance imaging predict for pathologic response to neoadjuvant chemotherapy in primary breast cancer. Clin Cancer Res 2008, 14(20):6580-6589.

21. Kuhl CK: Current status of breast MR imaging. Part 2. Clinical applications. Radiology 2007, 244(3):672-691.

22. Li KL, Henry RG, Wilmes LJ, Gibbs J, Zhu X, Lu Y, Hylton NM: Kinetic assessment of breast tumors using high spatial resolution signal enhancement ratio (SER) imaging. Magn Reson Med 2007, 58(3):572-581.

23. Li X, Rooney WD, Springer CS Jr: A unified magnetic resonance imaging pharmacokinetic theory: intravascular and extracellular contrast reagents. Magn Reson Med 2005, 54(6):1351-1359.

24. Kuhl CK, Schild HH, Morakkabati N: Dynamic bilateral contrast-enhanced MR imaging of the breast: trade-off between spatial and temporal resolution. Radiology 2005, 236(3):789-800.

25. Eyal $\mathrm{E}$, Degani $\mathrm{H}$ : Model-based and model-free parametric analysis of breast dynamic-contrast-enhanced MRI. NMR Biomed 2009.

26. van Osch MJ, Vonken EJ, Bakker CJ, Viergever MA: Correcting partial volume artifacts of the arterial input function in quantitative cerebral perfusion MRI. Magn Reson Med 2001, 45(3):477-485.

27. Weinmann HJ, Laniado M, Mutzel W: Pharmacokinetics of GdDTPA/ dimeglumine after intravenous injection into healthy volunteers. Physiol Chem Phys Med NMR 1984, 16(2):167-172.

28. Riethdorf S, Pantel K: Clinical relevance and current challenges of research on disseminating tumor cells in cancer patients. Breast Cancer Res 2009, 11(Suppl 3):S10.

29. Pierga JY, Bidard FC, Mathiot C, Brain E, Delaloge S, Giachetti S, de Cremoux P, Salmon R, Vincent-Salomon A, Marty M: Circulating tumor cell detection predicts early metastatic relapse after neoadjuvant chemotherapy in large operable and locally advanced breast cancer in a phase II randomized trial. Clin Cancer Res 2008, 14(21):7004-7010.

30. Sataloff DM, Mason BA, Prestipino AJ, Seinige UL, Lieber CP, Baloch Z: Pathologic response to induction chemotherapy in locally advanced carcinoma of the breast: a determinant of outcome. J Am Coll Surg 1995, 180(3):297-306.

31. Miller $A B$, Hoogstraten $B$, Staquet $M$, Winkler $A$ : Reporting results of cancer treatment. Cancer 1981, 47(1):207-214.

32. Port RE, Knopp MV, Brix G: Dynamic contrast-enhanced MRI using GdDTPA: interindividual variability of the arterial input function and consequences for the assessment of kinetics in tumors. Magn Reson Med 2001, 45(6):1030-1038.

33. Balvay D, Frouin F, Calmon G, Bessoud B, Kahn E, Siauve N, Clement O, Cuenod CA: New criteria for assessing fit quality in dynamic contrastenhanced T1-weighted MRI for perfusion and permeability imaging. Magn Reson Med 2005, 54(4):868-877.
34. Tofts PS, Berkowitz B, Schnall MD: Quantitative analysis of dynamic GdDTPA enhancement in breast tumors using a permeability model. Magn Reson Med 1995, 33(4):564-568.

35. DeLong ER, DeLong DM, Clarke-Pearson DL: Comparing the areas under two or more correlated receiver operating characteristic curves: a nonparametric approach. Biometrics 1988, 44(3):837-845

36. Wolmark N, Wang J, Mamounas E, Bryant J, Fisher B: Preoperative chemotherapy in patients with operable breast cancer: nine-year results from National Surgical Adjuvant Breast and Bowel Project B-18. J Natl Cancer Inst Monogr 2001, , 30: 96-102.

37. Sevick EM, Jain RK: Geometric resistance to blood flow in solid tumors perfused ex vivo: effects of tumor size and perfusion pressure. Cancer Res 1989, 49(13):3506-3512

38. Sevick EM, Jain RK: Viscous resistance to blood flow in solid tumors: effect of hematocrit on intratumor blood viscosity. Cancer Res 1989, 49(13):3513-3519.

39. Less JR, Skalak TC, Sevick EM, Jain RK: Microvascular architecture in a mammary carcinoma: branching patterns and vessel dimensions. Cancer Res 1991, 51(1):265-273.

40. Li SP, Taylor NJ, Makris A, Ah-See ML, Beresford MJ, Stirling JJ, d'Arcy JA, Collins DJ, Padhani AR: Primary human breast adenocarcinoma: imaging and histologic correlates of intrinsic susceptibility-weighted MR imaging before and during chemotherapy. Radiology 257(3):643-652.

41. Wedam SB, Low JA, Yang SX, Chow CK, Choyke P, Danforth D, Hewitt SM, Berman A, Steinberg SM, Liewehr DJ, Plehn J, Doshi A, Thomasson D, McCarthy N, Koeppen H, Sherman M, Zujewski J, Camphausen K, Chen H, Swain SM: Antiangiogenic and antitumor effects of bevacizumab in patients with inflammatory and locally advanced breast cancer. J Clin Oncol 2006, 24(5):769-777.

42. Green AJ, Francis RJ, Baig S, Begent RH: Semiautomatic volume of interest drawing for (18)F-FDG image analysis-method and preliminary results. Eur J Nucl Med Mol Imaging 2008, 35(2):393-406.

43. Chen W, Giger ML, Bick U: A fuzzy c-means (FCM)-based approach for computerized segmentation of breast lesions in dynamic contrastenhanced MR images. Acad Radiol 2006, 13(1):63-72.

44. Stoutjesdijk MJ, Veltman J, Huisman H, Karssemeijer N, Barentsz JO, Blickman JG, Boetes C: Automated analysis of contrast enhancement in breast MRI lesions using mean shift clustering for ROI selection. J Magn Reson Imaging 2007, 26(3):606-614.

45. Henderson E, Rutt BK, Lee TY: Temporal sampling requirements for the tracer kinetics modeling of breast disease. Magn Reson Imaging 1998, 16(9):1057-1073.

46. Li SP, Makris A, Beresford MJ, Taylor NJ, Ah-See ML, Stirling JJ, d'Arcy JA, Collins DJ, Kozarski R, Padhani AR: Use of Dynamic Contrast-enhanced MR Imaging to Predict Survival in Patients with Primary Breast Cancer Undergoing Neoadjuvant Chemotherapy. Radiology 2011, 260(1):68-78.

47. Li SP, Padhani AR, Taylor NJ, Beresford MJ, Ah-See ML, Stirling JJ, d'Arcy JA, Collins DJ, Makris A: Vascular characterisation of triple negative breast carcinomas using dynamic MRI. Eur Radiol 2011, 21(7):1364-1373.

48. Li KL, Wilmes LJ, Henry RG, Pallavicini MG, Park JW, Hu-Lowe DD, McShane TM, Shalinsky DR, Fu YJ, Brasch RC, Hylton NM: Heterogeneity in the angiogenic response of a BT474 human breast cancer to a novel vascular endothelial growth factor-receptor tyrosine kinase inhibitor: assessment by voxel analysis of dynamic contrast-enhanced MRI. J Magn Reson Imaging 2005, 22(4):511-519.

49. Planey CR, Welch EB, Xu L, Chakravarthy AB, Gatenby JC, Freehardt D, Mayer I, Meszeoly I, Kelley M, Means-Powell J, Gore JC, Yankeelov TE: Temporal sampling requirements for reference region modeling of DCEMRI data in human breast cancer. J Magn Reson Imaging 2009, 30(1):121-134.

50. Pinker K, Grabner G, Bogner W, Gruber S, Szomolanyi P, Trattnig S, HeinzPeer G, Weber M, Fitzal F, Pluschnig U, Rudas M, Helbich T: A combined high temporal and high spatial resolution 3 Tesla MR imaging protocol for the assessment of breast lesions: initial results. Invest Radiol 2009, 44(9):553-558.

51. Tofts PS: Modeling tracer kinetics in dynamic Gd-DTPA MR imaging. J Magn Reson Imaging 1997, 7(1):91-101.

52. Daldrup HE, Shames DM, Husseini W, Wendland MF, Okuhata Y, Brasch RC: Quantification of the extraction fraction for gadopentetate across breast cancer capillaries. Magn Reson Med 1998, 40(4):537-543. 
53. Wasser K, Sinn HP, Fink C, Klein SK, Junkermann H, Ludemann HP, Zuna I, Delorme S: Accuracy of tumor size measurement in breast cancer using MRI is influenced by histological regression induced by neoadjuvant chemotherapy. Eur Radiol 2003, 13(6):1213-1223.

54. Denis F, Desbiez-Bourcier AV, Chapiron C, Arbion F, Body G, Brunereau L: Contrast enhanced magnetic resonance imaging underestimates residual disease following neoadjuvant docetaxel based chemotherapy for breast cancer. Eur J Surg Oncol 2004, 30(10):1069-1076.

55. Rieber A, Zeitler H, Rosenthal H, Gorich J, Kreienberg R, Brambs HJ, Tomczak R: MRI of breast cancer: influence of chemotherapy on sensitivity. Br J Radiol 1997, 70(833):452-458.

56. Kuhl CK, Traber F, Gieseke J, Drahanowsky W, Morakkabati-Spitz N Willinek W, von Falkenhausen M, Manka C, Schild HH: Whole-body highfield-strength (3.0-T) MR imaging in clinical practice. Part II. Technical considerations and clinical applications. Radiology 2008, 247(1):16-35.

57. Connett JE, Smith JA, McHugh RB: Sample size and power for pairmatched case-control studies. Stat Med 1987, 6(1):53-59.

58. Danishad KK, Sharma U, Sah RG, Seenu V, Parshad R, Jagannathan NR: Assessment of therapeutic response of locally advanced breast cancer (LABC) patients undergoing neoadjuvant chemotherapy (NACT) monitored using sequential magnetic resonance spectroscopic imaging (MRSI). NMR Biomed 2010, 23(3):233-241.

59. Park SH, Moon WK, Cho N, Song IC, Chang JM, Park IA, Han W, Noh DY: Diffusion-weighted MR imaging: pretreatment prediction of response to neoadjuvant chemotherapy in patients with breast cancer. Radiology 2010, 257(1):56-63

\section{Pre-publication history}

The pre-publication history for this paper can be accessed here: http://www.biomedcentral.com/1471-2407/11/361/prepub

doi:10.1186/1471-2407-11-361

Cite this article as: de Bazelaire et al:: Accuracy of perfusion MRI with high spatial but low temporal resolution to assess invasive breast cancer response to neoadjuvant chemotherapy: a retrospective study. BMC Cancer 2011 11:361.

\section{Submit your next manuscript to BioMed Central and take full advantage of:}

- Convenient online submission

- Thorough peer review

- No space constraints or color figure charges

- Immediate publication on acceptance

- Inclusion in PubMed, CAS, Scopus and Google Scholar

- Research which is freely available for redistribution

Submit your manuscript at www.biomedcentral.com/submit 